\title{
New Idea to Enhance Better Understanding of Free Body Diagrams in Solid Mechanics Course
}

\author{
Raghu Echempati \\ Department of Mechanical Engineering, Kettering University, Flint, MI - 48504 (USA) \\ rechempa@kettering.edu
}

\begin{abstract}
Historically, majority of students taking solid mechanics course at my university find it confusing and even challenging to draw free body diagrams (FBDs) and to compute the internal forces correctly. Many instructors teach those topics in typical statics course and review the same principles in solid mechanics and design courses. However, many students either do not still fully understand the topic or have forgotten the concepts during the time gap between taking statics and the follow on courses. This is also true for colleges such as ours with mandatory co-op education in which students alternate between school and work terms. With incorrect calculation of internal loads (or load in each section of a member), the next steps of calculating the stress and deflection of members will go wrong. In a recent solid mechanics course taught by the author, 60 to 70 percent of the students could not correctly compute the internal forces within each section of an axially loaded member. Although majority of students drew the free body diagram of the entire system correctly, only a few could realize or interpret the correct values of forces within each section of a member. Others drew incorrect FBDs that do not satisfy static equilibrium.
\end{abstract}

\section{Raghu Echempati}

Department of Mechanical Engineering, Kettering University, Flint, MI - 48504 (USA) rechempa@kettering.edu
The author came up with an idea (alternative approach or method) to teach free body diagrams that partially address the above-mentioned difficulties that many students face. Similar to axial loading, students find it difficult to determine the internal loads for torsional members. Determining the internal loads for beams subjected to complex loading is even more confusing and challenging to these students. Although the new method proposed in this paper is not fully assessed for its effectiveness, it was used during the Fall 2018 (September to December) and Winter 2019 (January to March) solid mechanics classes. The total number of students was around 50 in both academic terms. The students viewed this new method as one of the potential approaches to understand the equilibrium concepts. Same course will be taught by the author during Spring 2019 term to assess the effectiveness of the proposed method 'before and after' by giving the same problems to students using the old approach and to students using the new approach. Preliminary assessment results based on the students' performance on in-class, homework, quizzes and exams will be available by the end of Winter 2019 term. Active learning activities are being used to help teach the method. It is hoped that this alternative method will be an effective approach for instructors teaching statics and solid mechanics courses to make the struggling students better understand the concepts of static equilibrium. Details of this approach will be discussed in detail in this paper along with several numerical examples.

Keywords: ABET, Static equilibrium in 2D, free body diagrams (FBDs), internal loads 


\section{Background Information And Review Of Literature:}

Numerous textbooks, educational papers and online videos are available on teaching and better understanding by the students of FBDs in statics and solid mechanics courses. MIT, Georgia Tech, Carnegie Mellon, and many other schools in USA and elsewhere offer free online courseware on this and other engineering courses [for example, see references 1 to 4]. Similarly, numerous textbooks such as Beer and Johnston, Hibbler, Goodno and Gere, etc., provide many worked examples to cover concepts of equilibrium and free body diagrams in great depth [see references 5 to 7 for example]. The author used Beer and Johnston's book for the Solid Mechanics course. McCarthy and Goldfinch described their method of designing questions on free body diagrams that address the visual learner and the equations of equilibrium [8]. Keeping in view the common misconceptions and mistakes of the students, they outlined some strategies for teaching the correct approach.

Many papers and posters have been presented or published in ASEE (American Society for Engineering Education) and other related educational conferences and journals on both assessment and effectiveness of teaching critical topics in Statics and Solid mechanics courses (mainly covering the topics on free body diagrams, internal loads, stress, and strain analysis) $[9,10]$. Since the intent of this paper is to outline the new idea and the effectiveness on students' better understanding of free body diagrams to determine the internal loads, detailed literature survey is not undertaken. The proposed idea or method is based on some intuition and satisfying the equations of equilibrium in a simple but step by step manner. The students were first taught the similarities and differences between terms: Applied, Reaction, External and Internal loads. Many students admitted to have learned this first time.

\section{A. Example of a Concept Application Problem 2.1 from Beer and Johnston [4]}

In order to begin a discussion on the new idea (method) presented in this paper, the author discussed the worked example from the textbook on axial loading of the member as shown in Fig. 1(a). The goal is to determine the stress and deformation in each portion of the bar, given the geometry, load and material of the bar (the 3 design variables). The associated free body diagrams reproduced from the book are shown in Figs. 1(b and c).

Majority of the students said that they are familiar with the way the free body diagrams were presented in Figures 1 (a) through (c), since they are similar to how the problems were solved in their Statics course. Before the new idea was presented, the students were asked to close the book and redraw free body diagrams for each segment of the same bar.

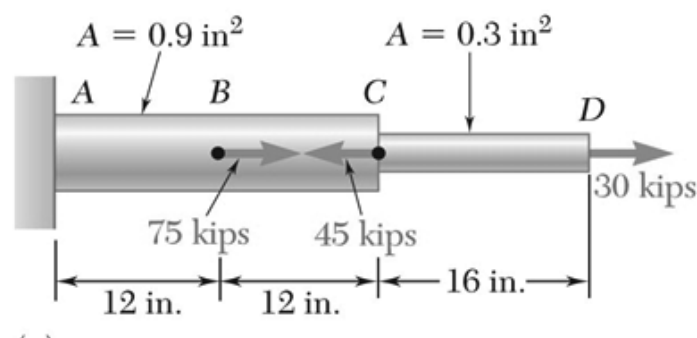

(a)

Fig. 1(a): Sketch of an axially-loaded bar with geometry and loads [4]

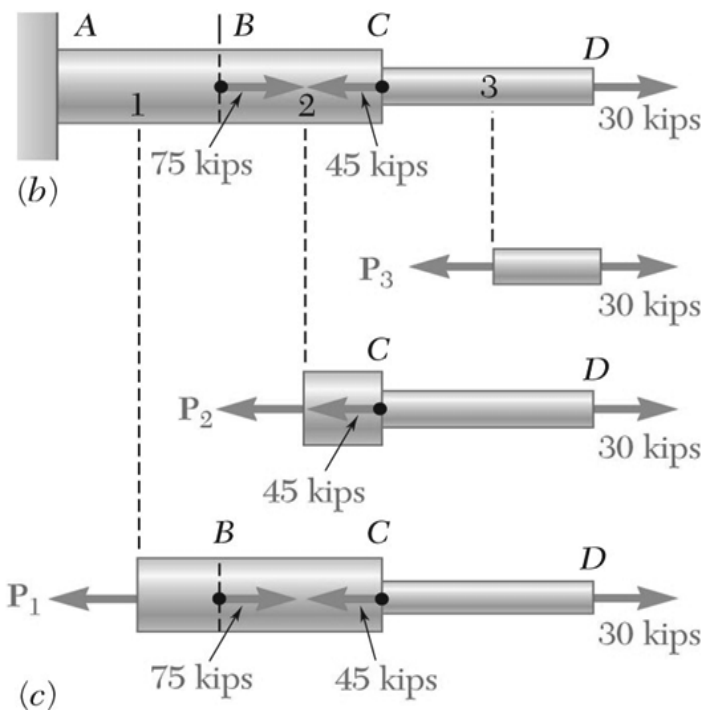

Fig. 1(b and c): Step by step sketches of the free body diagrams [4]

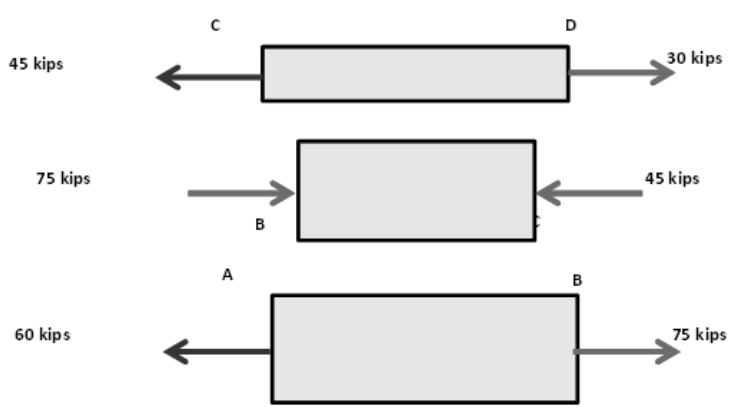

Fig. 2: Common mistakes of students while drawing the FBDs of Figure 1 
However, majority of them had difficulty to realize the forces in each segment (AB, BC and CD) of the bar in this example. Consequently, only a few (less than $50 \%$ ) drew correct free body diagrams for each segment of the bar to determine the loads. Rest of the students did a variety of mistakes, some of which are shown in Fig. 2. It is the author's belief that understanding the proper meaning of the word 'static equilibrium' requires some maturity and a 'feel' for it. More confusing is that while statics deals with 'rigid bodies', solid mechanics deals with 'deformable bodies. So the challenge to the young minds is to understand what a rigid body means and how to quantify it. It is not that the instructors do not explain thiese, but it is do with the maturity level of the students to understand the basic physics and physical laws of equilibrium.

At the beginning of the topic on rigid body equilibrium, we often mention or assume that selfweight of a rigid body $(\mathrm{W}=\mathrm{m} * \mathrm{~g})$ is neglected, whereas in physics course they learned Newton's 2nd law of motion in which gravitational effects are discussed. Even if we explain the rationale for neglecting the self-weight of the bodies (that in comparison to the magnitude of the applied loads, the self-weight is 'negligible'), the young minds do not seem to grasp it properly. Most students understand the math behind the equations of equilibrium but do not understand the difference between external and internal loads. To some degree or the other, these or similar other mistakes are commonly done by the students in the follow on courses at other institutions as well, and most instructors (should) have an experience of this. Thus, it is the poor understanding of the concept of the words 'static equilibrium'. Confusion about the concept of 'dynamic equilibrium' is another topic.

Computing the internal loads for section $\mathrm{BC}$ turned out to be the most difficult for the majority of students who did the mistakes. It may be noted that not all students (whose work was wrong) did all these mistakes. Also, there were other kinds of errors they made that are not shown here. It is clear from these mistakes, that the students lack a basic understanding of the equilibrium concepts. Some students are confused that equilibrium is not maintained since the body 'deforms'. This is perhaps due to the 'rigid body' assumption that we make in Statics course. As mentioned earlier, these confusions and misconceptions were mostly due to a lack of understanding of the words "equilibrium" and "internal versus external loads" by many students.

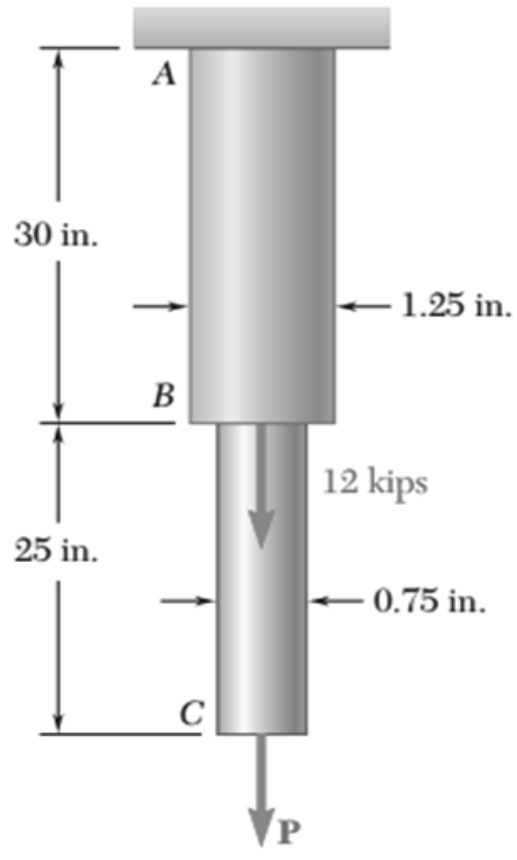

Fig. 3: Sketch of the stepped bar with intermediate loads [5]

The other challenge is the management of time to complete the problem. In a typical statics course drawing detailed free body diagrams such as those shown in Figure 1 to compute the internal loads is a problem by itself. However, for solid mechanics course, students should take less time to complete the statics portion of the problem, and continue on to determine the stress and deflection parts of the question in solid mechanics.

After these concepts are clarified by the author during the lecture, and a second simpler problem was given to them to solve, quite a few still could not correctly draw free body diagrams. They didn't seem to still understand or retain what was taught to them in the prerequisite classes. This was in spite of practicing problems in the class as a group, and also submitting the assigned homework. Typically, the students at our university take 4 to 5 different (4-credit) courses in a term (i.e., 16 to 20 hours of classes per week), which may be one of the reasons for managing their time between all courses. We also provide tutoring or help sessions at our university, which are usually meagerly attended, again perhaps due to their heavy course load.

Initially, a question similar to the following example was given on the first Quiz before the proposed new idea or method was taught. More than $70 \%$ of the students of the class drew wrong free body 


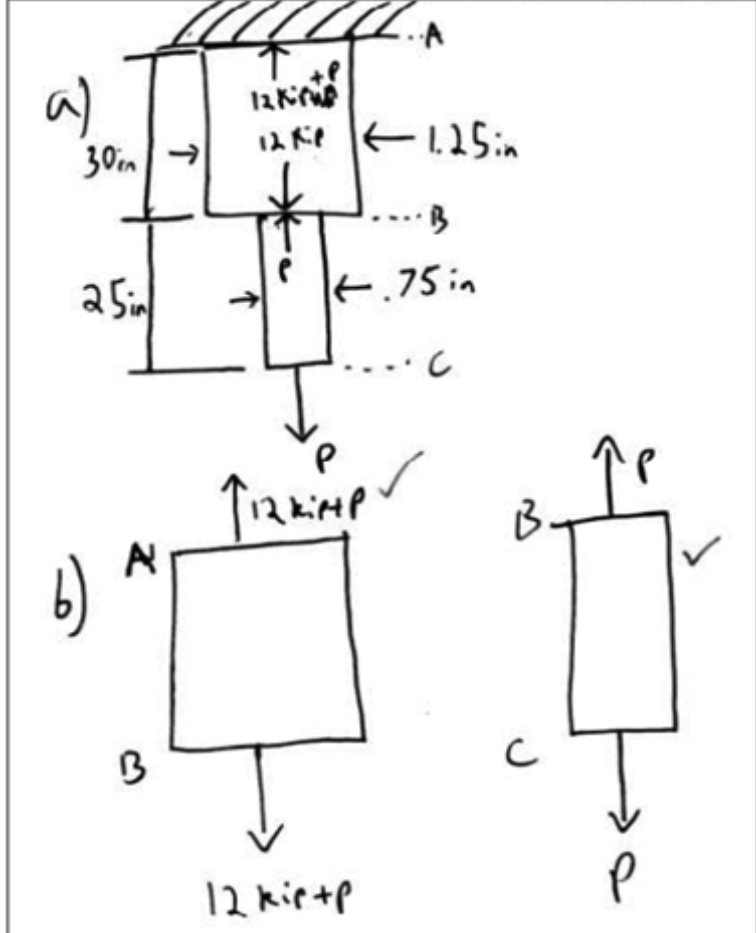

Fig. 4: Sample free body diagrams drawn correctly

diagrams that lead to incorrect answers for remaining parts of this question. Later for Midterm Exam 1, the following question (Fig. 3) was given which is based on statics (equilibrium, free body diagrams) and solid mechanics (stress, deformation, strain, and Poisson's ratio). The total number of points for this question was 25 . By this time, the students were taught the proposed new method of solving for internal loads (discussed later). However, $20 \%$ of the students still made one mistake or the other in computing the internal forces. The statement of the multiple-part question is as follows, which is a modified version of the exercise problem from Beer and Johnston book [5]. This is a statically determinate problem.

Two solid cylindrical rods $\mathrm{AB}$ and $\mathrm{BC}$ are welded together at B and loaded as shown in Figure 3. (a) Draw free body diagram of the entire system, (b) Free body diagrams of portions $\mathrm{AB}$ and $\mathrm{BC}$ (in terms of the unknown force, P), (c) Determine the magnitude of the force $\mathrm{P}$ for which the tensile stress in $\operatorname{rod} \mathrm{AB}$ is 1.6 times that in rod $\mathrm{BC}$, (d) Knowing the value of $\mathrm{P}$ from part (c), determine the deformation and linear strain in sections $\mathrm{AB}$ and $\mathrm{BC}$, (e) Determine the original volume of portion $\mathrm{BC}$ (prior to loading), (f) Assuming that the volume of that portion after loading is the same as the original volume, find the reduction in diameter of BC, and (g) Determine the Poisson's ratio for section $\mathrm{BC}$.
This is a multiple-part, multi-step question, with each part depending on determining correctly the internal loads. As per the rubrics, maximum weightage was given to statics portion, Units, and using the correct equations based on the given data to obtain correct internal load values. This will ensure that the other parts are correctly solved.

Correct answer to free body diagrams and the several parts of the question were obtained by 23 out of 36 students (about 64\%). Sample students' free body sketches are shown in Figure 4. Figure 5 shows an example of incorrectly drawn free body diagrams that lead to incorrect answers to rest of the parts of the question.

2. Discussion Of The Proposed New Ideas (approaches) For Free Body Diagrams

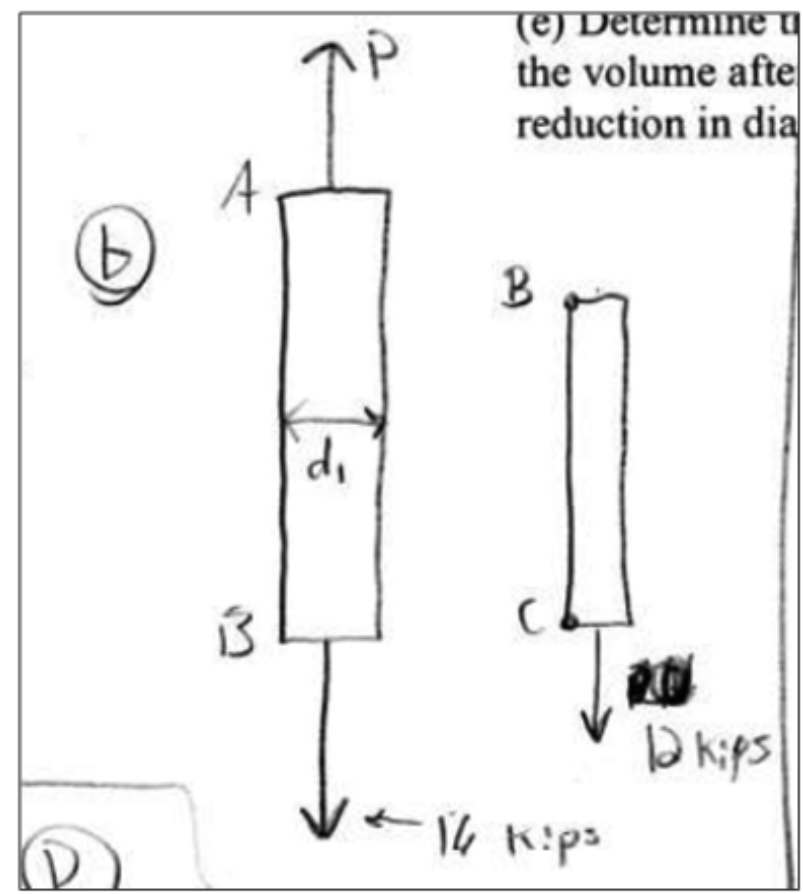

Fig. 5: Sample free body diagrams drawn incorrectly

A. Verification of internal loads from free body diagrams:

When the students were clear about understanding the similarities and difference between the external applied loads, reaction loads and internal loads, they were asked to verify that the free body diagrams drawn for each portion of the bar along with the calculated internal loads when 'put back together', should yield the same externally applied load at that 


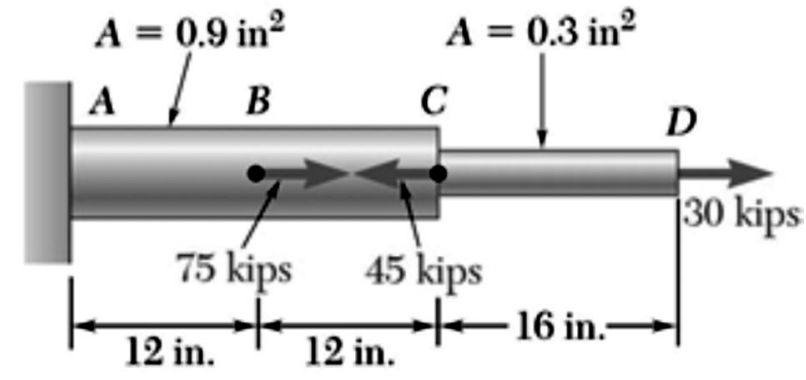

(a)

Fig. 1(a): Original sketch of an axially-loaded bar with geometry and external loads [5]

particular location (section) as on the original bar. As an example, refer to the problem shown in Fig. 1(a) (reproduced below). The internal (reaction) load at section $\mathrm{C}$ of portion $\mathrm{CD}$ of the bar equals to $30 \mathrm{kips}$ (to the left, for equilibrium), and the internal load at same section $\mathrm{C}$ of portion $\mathrm{BC}$ of the bar equals to $15 \mathrm{kips}$ (to the left, for equilibrium), as shown in Figure 6. This means that portion $\mathrm{CD}$ is in tension while portion $\mathrm{BC}$ is in compression. Since in reality the bar is one-piece with $\mathrm{CD}$ and $\mathrm{BC}$ joined at $\mathrm{C}$, the corresponding free body diagrams along with the calculated internal loads for these two portions when 'merged together', should yield 'the same loading situation' as the original bar for those portions. This is true since at section $\mathrm{C}$, the internal forces - 30 kips (for CD) and - 15 kips (for BC) upon 'merging', yield the originally applied (external) load of - 45 kips (i.e., to the left). Students were also asked to notice that upon merging portions $\mathrm{BC}$ and $\mathrm{CD}$ at $\mathrm{C}$, the portion $\mathrm{BCD}$ of the bar still maintains static equilibrium (sum forces in $\mathrm{x}$ direction is still equal to zero). Same procedure can be followed for section B.
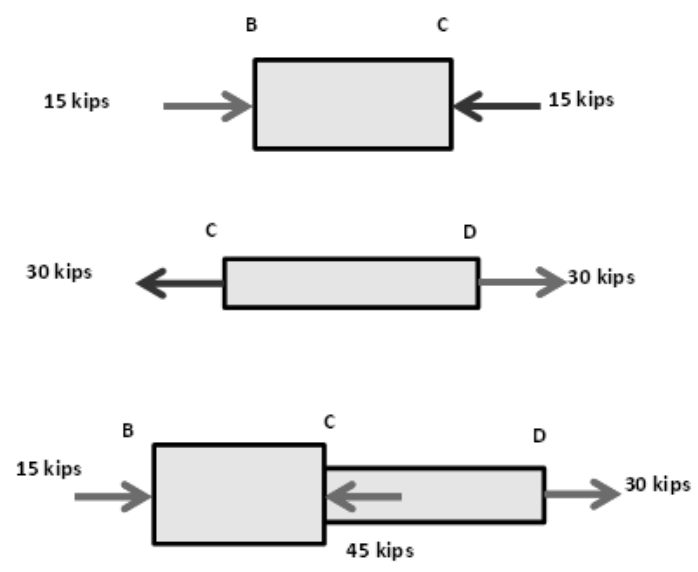

Fig. 6: Individual free body diagrams for portions $B C$ and CD (above two), and for portion BCD of the bar (bottom figure)
Almost all students of the class indicated that this is a very helpful new technique for them to better understand the free body diagrams. Perhaps they were taught using the traditional or conventional method similar to Figure 1(b and c), shown previously that is normally presented in many Statics and Solid Mechanics textbooks, which is okay. However, transforming that knowledge to solve Solid mechanics problems seem to be not obvious to many students with the result that they are confused to understand that the free body diagrams shown in Fig. $1(\mathrm{~b}, \mathrm{c})$, provide the same information to determine the internal loads as detailed in Figure 6. Also, many instructors perhaps teach free body diagrams using both ways. Still, why many students do these mistakes? At our university, the follow-on Machine Design course is taken 6 months or more after the Solid mechanics course was taken (due to mandatory co-op term(s), and other scheduling issues), with the result that the instructor has to re-teach (not just a simple review of) free body diagrams and the concepts of static equilibrium.

A. Intuitive approach to help better understand the free body diagrams

The author used another simpler idea or approach (or method) to draw free body diagrams. This is based on intuitive approach. As an example, students were given the sketch of a bar (unbalanced) with a single intermediate load applied at section $\mathrm{C}$, as shown in Figure 7(a). In step 1 (Figure 7(b)), they were asked to draw sketches of the two sections of the bar where the $5,000 \mathrm{~N}$ load is applied, but show no loads on them yet. In this case, it is at section $\mathrm{C}$ of the bar (with no loads shown in either section). In step 2 (Figure 7(c)), they were asked to give any two numbers (force

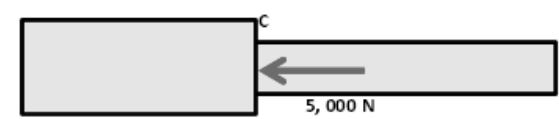

(a): Original diagram
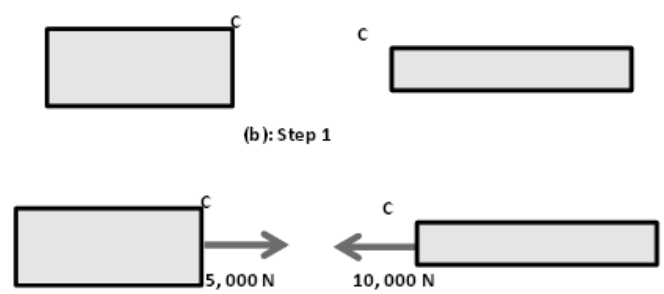

(c): Step 2

Fig. 7: Step by step procedure in the "Intuitive Method" of drawing equivalent force system at section $\mathrm{C}$ of the bar 
values) with proper directions such that when the two sections are 'joined' together at $\mathrm{C}$, would yield the same force (magnitude and direction) as the original applied load. Obviously, there are infinite possible solutions to this idea. One set of force values is shown in Figure $7(\mathrm{c})$ that are statically equivalent to the original figure. Same logic can be applied when two bars made of different materials are joined at a section (such as C).

Figure 8 shows the same example (of an unbalanced bar) but with an additional force acting at section D of the bar. Following the same logic, in step 1 , students are asked to draw two sketches of the divided bar with no loads shown on them. In step 2, they are asked to start from right end, and show the external load acting at D of the bar. In the next step, they are asked to first provide the internal load at $C$ on the left to keep the portion CD of the bar in static equilibrium. With this, in the final step, they are asked to determine the force (magnitude and direction) at section $\mathrm{C}$ of portion $\mathrm{BC}$ of the bar such that when these two portions ( $\mathrm{BC}$ and $\mathrm{CD}$ ) along with the two force vectors are 'joined back together' would yield the originally applied force of 5, $000 \mathrm{~N}$. In this example, it becomes obvious to the students that there is only one solution to the problem that satisfies the static equilibrium conditions for portion $\mathrm{CD}$, and at section $\mathrm{C}$ of on both sides of the bar. In this example, if section $\mathrm{B}$ were to be a fixed end, then $\mathrm{BC}$ will experience compression (load of $1,200 \mathrm{~N}$ ), and CD will experience tension load of $3,800 \mathrm{~N}$.

Almost all students of the class that the author taught in two successive terms appreciated this step by step 'dissected method' of drawing of free body
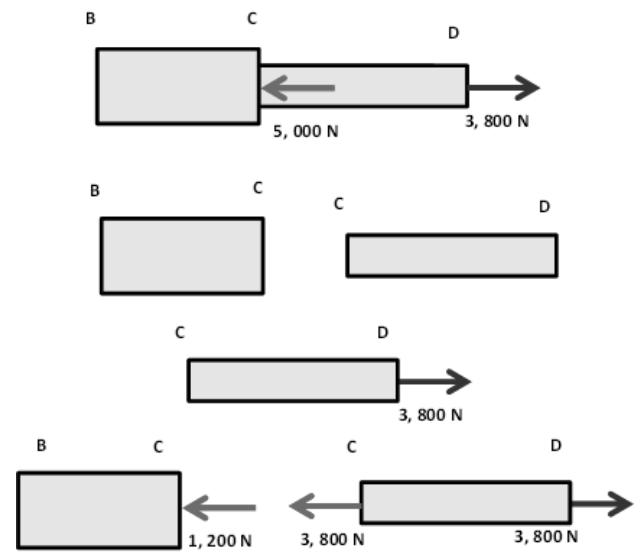

Fig. 8: Additional example demonstrating the "Intuitive Method" of drawing equivalent force system of a partially-loaded bar diagrams. Those $30 \%$ - $40 \%$ of the students who drew wrong free body diagrams before these methods were actually practiced in the one-hour of the class, did much better on the quizzes and exams, although not all of them could solve actual exercise problems correctly.

The intuitive method presented in this paper may help some students with a different learning style in better understanding the calculation of internal loads of axial and torsion members by drawing correct free body diagrams. However, students are cautioned that they may not have enough time on the quizzes and exams to draw detailed step by step free body diagrams as explained in this paper. There will be follow-on parts of the problems to do stress and deflection analyses. They must master the equilibrium concepts by practicing more homework problems and realizing how internal loads at each section when 'joined back' would yield the same externally applied load at that section. Although drawing free body diagrams sounds easy for the instructors, students seem to not feel that way due to not correctly understanding the concepts of equilibrium. Calculating internal loads for beams in bending is still more challenging compared to axial and torsional loadings.

The alternative method presented in this paper may help instructors explain these concepts better to those who struggle in the classes. Discussing real life examples and practical applications in the form of mini-projects also help them in better understanding of the concepts of Statics and equilibrium equations.

One of the real life examples of a bar loaded at multiple sections that the author uses is shown in

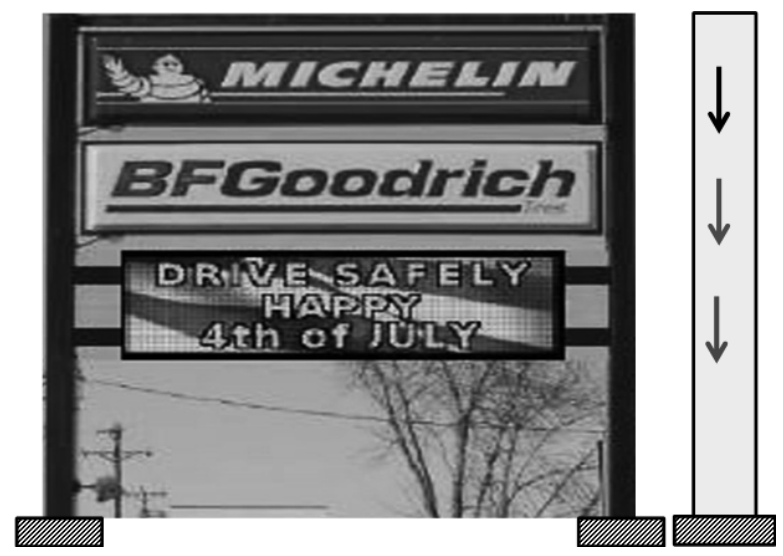

Fig. 9: Real life example of a frame with multiple sign boards (left), and the simplified axial loading of an 'equivalent bar' with multiple intermediate loads 
Figure 9 of multiple signs or banners attached to a single frame. Although not completely correct, this can be reduced to a simplified rough model with loads acting only in the vertical direction due to weight of each sign board as shown in Figure 9. Obviously, wind loads can cause bending and torsion loads in addition to axial loads on the frame, and a detailed frame analysis is needed to design such structures.

The author discusses many such real life examples and applications in this, and in other courses using textbook problems that are only conceptual in nature. Additionally, students are involved in active learning by discussing the safety issues if these are poorly designed and how it may impact the society at large (loss of jobs, transportation delays, etc.). So, some emphasis is put on the discussion of the results obtained for a particular problem and how the results vary if some of the design parameters are changed.

\section{Conclusions}

In this paper a new strategy to explain the concepts of static equilibrium using free body diagrams is presented through simple examples and explanations of an axially-loaded bar. These ideas can be adopted in Statics course when teaching the internal loads topics on torsion and bending. Also, the equilibrium concepts explained here can be easily applied to statically determinate problems. Once this is understood by the students, similar ideas and strategies can be successfully extended to statically indeterminate problems in Solid Mechanics involving thermal stresses, with or without additional external loads applied at intermediate sections of a bar or a beam.

Sample students' work on free body diagrams for axially-loaded bars is presented that show where students commonly make the familiar mistakes. The class performance (on their homework, quizzes and exams) before this strategy was adopted showed that more than $50 \%$ of the class did not understand the equilibrium concepts. Part of the reason for this although may be attributed (at this university) to the gaps between the academic terms (due to co-op) and retention of knowledge, such students still were confused due to insufficient understanding of the topic on internal loads when they took Statics course. After the new method or the idea presented in this paper was adopted in the class, it helped majority of the students to better understand the internal loads and to perform well on the final exams. It is believed that this new method of explanation of free body diagrams may be useful to instructors at other colleges. This may lead to evolution of other new ideas of teaching techniques to the Mechanics Community.

\section{References}

[1] https://ocw.mit.edu/courses/mechanicalengineering/2-001-mechanics-materials-i-fall2006/lecture-notes/

[2] Massachusetts Institute of Technology (MIT) Open Courseware (2001 to 2020) available at: https://ocw.mit.edu/courses/materials-scienceand-engineering/3-11-mechanics-of-materialsfall-1999/modules/

[3] Georgia Institute of Technology (GIT) Courseware (2018 to 2020), "Module 23: Free Body Diagrams I", available at: https://www.coursera.org/lecture/engineeringmechanics-statics/module-23-free-bodydiagrams-i-JnLmx

[4] Statics OLI at Carnegie Mellon University (CMU) Course Modules (2020) available at: https://oli.cmu.edu/courses/engineering-staticsopen-free/

[5] F. P. Beer, E. R. Johnston Jr., J. T. DeWolf, and D. F. Mazurek, Mechanics of Materials, 7th Edition, published by McGraw-Hill, 2017.

[6] R. C. Hibbler. Mechanics of Materials, 10th Edition, Pearson Publishers, 2017.

[7] B. J. Goodno, and J. M. Gere. Mechanics of Materials, 9th Edition, PWS Kent Publishers, 2018.

[8] T. J. McCarthy and T. Goldfinch, "Teaching the concept of free body diagrams", Proceedings of the 2010 AaeE Conference, Sydney, 2010.

[9] Shih-Liang Wang, "Free Body Diagrams with Animated GIF Files" Paper ID \#16401, Proceedings of 2016 ASEE Conference, New Orleans, LA, 2016.

[10] K. N. Leipold and S. R. Ivancic, "Efforts to Improve Free Body Diagrams", Paper ID \# 22338, Proceedings of 2018 ASEE Conference, Salt Lake City, UT, 2018. 